\title{
CLINICAL SIGNIFICANCE OF GENERALIZED SPIKE/WAVE EEG
}

The clinical correlates of 154 children with epilepsy and generalized spikeand-wave (SW) EEG patterns were analyzed at the pediatric neurology units, HU de Getafe and Hospital 12 de Octubre, Madrid, Spain. Mean age at seizure onset was 6 years (range, 0 - 14 years). Eighteen percent had learning disorders, and 14\% had a history of febrile seizures. Ten percent of first-degree relatives had epilepsy. Absence seizures were the most frequent type at onset (36\%); tonic-clonic seizures occurred in 27\%, and complex partial seizures in $14 \%$. More than $40 \%$ had several types of seizures at time of evaluation: $48 \%$ had at least one tonic-clonic, $46 \%$ absence, 20\% complex partial. Epilepsy syndrome diagnoses included idiopathic generalized epilepsy (49\%), and partial epilepsies (24\%). The typical SW EEG pattern was usually associated with a single type of seizure, mainly idiopathic absence, normal neurologic exam and CT scan, no learning problems, and favorable outcome with monotherapy, sustained after drug withdrawal. Slow SW correlated with more than one type of seizure (atonic, myoclonic, tonic, and partial), West syndrome, psychomotor retardation, abnormal CT, polytherapy and poor seizure control. Fast $S W$ occurred with partial and secondary generalized seizures, normal CT, and seizure recurrence after drug withdrawal. (MartinezMenendez B, Sempere AP, Mayor P, et al. Generalized spike-and-wave patterns in children: clinical correlates. Pediatr Neurol Jan 2000; 22:23-28). (Respond: Dr Martinez-Menendez, U Neuropediatria, S Neurologia, HU Getafe, Ctra de Toledo Km 12.400, 28905-Gefate, Madrid, Spain).

COMMENT. The typical SW EEG pattern associated with absence seizures has a much more benign outcome than the slow SW, usually associated with West and Lennox Gastaut syndromes. In contrast, the clinical course of children with the fast SW EEG is more difficult to predict, being associated with single isolated seizures and a tendency to seizure recurrence. Typical SW is 10 times more likely to correlate with seizure remission and successful drug withdrawal than the slow SW pattern.

\section{MECHANISM OF THE KETOGENIC DIET}

To elucidate the possible significance of ketone bodies in the mechanism of the ketogenic diet, researchers at Washington University School of Medicine, St Louis, MO, examined the effect of B-hydroxybutyrate and acetoacetate on excitatory and inhibitory synaptic transmission and spontaneous seizures in rat hippocampal slices and cultured hippocampal neurons. Ketone bodies had no effect on synaptic transmission in these models, and the hypothesis that ketone production is involved in the anticonvulsant action of the diet was not substantiated. (Thio LL, Wong M, Yamada KA. Ketone bodies do not directly alter excitatory or inhibitory hippocampal synaptic transmission. Neurology January (2 of 2) 2000;54:325-331). (Reprints: Dr Kelvin A Yamada, Washington University School of Medicine, Department of Neurology, Box 8111, 660 S Euclid Ave, St Louis, MO 63310).

COMMENT. Stafstrom CE and Spencer S, in an editorial comment, suggest that further studies on the possible anticonvulsant effects of ketones should be conducted in animals of different ages and after chronic ketogenic diet treatment (Neurology Jan 2000;54:282-283). Perhaps further studies involving electrolyte changes induced by the diet would be more productive.

An electrolyte balance study in children with absence seizures treated with the ketogenic diet showed decreases in the blood $\mathrm{pH}, \mathrm{PCO} 2$, and standard bicarbonate during ketosis (Millichap JG, Jones JD. Acid-base, electrolyte, and 